\title{
A Comparison of Usage and Outcomes Between Nurse Practitioner and Resident-Staffed Medical ICUs.
}

\author{
Rachel Scherzer \\ Thomas Jefferson University \\ Marie P Dennis \\ Thomas Jefferson University \\ Beth Ann Swan \\ Thomas Jefferson University \\ Mani Kavuru \\ Thomas Jefferson University \\ David A. Oxman \\ Thomas Jefferson University \\ Follow this and additional works at: https://jdc.jefferson.edu/nursfp \\ Part of the Nursing Commons \\ Let us know how access to this document benefits you
}

\section{Recommended Citation}

Scherzer, Rachel; Dennis, Marie P; Swan, Beth Ann; Kavuru, Mani; and Oxman, David A., "A Comparison of Usage and Outcomes Between Nurse Practitioner and Resident-Staffed Medical ICUs." (2017). College of Nursing Faculty Papers \& Presentations. Paper 84.

https://jdc.jefferson.edu/nursfp/84

This Article is brought to you for free and open access by the Jefferson Digital Commons. The Jefferson Digital Commons is a service of Thomas Jefferson University's Center for Teaching and Learning (CTL). The Commons is a showcase for Jefferson books and journals, peer-reviewed scholarly publications, unique historical collections from the University archives, and teaching tools. The Jefferson Digital Commons allows researchers and interested readers anywhere in the world to learn about and keep up to date with Jefferson scholarship. This article has been accepted for inclusion in College of Nursing Faculty Papers \& Presentations by an authorized administrator of the Jefferson Digital Commons. For more information, please contact: JeffersonDigitalCommons@jefferson.edu. 


\title{
A Comparison of Usage and Outcomes Between Nurse Practitioner and Resident-Staffed Medical ICUs
}

\author{
Scherzer, Rachel MSN, RN, ACNP-BC; Dennis, Marie P. PhD, EdM; Swan, Beth \\ Ann PhD, CRNP, FAAN; Kavuru, Mani S. MD; Oxman, David A. MD, FACP
}

Author Information

${ }^{1}$ Thomas Jefferson University, Jefferson College of Nursing, Philadelphia, PA.

${ }^{2}$ Division of Pulmonary and Critical Care Medicine, Thomas Jefferson University, Philadelphia, PA.

This work was performed at Thomas Jefferson University, Philadelphia, PA.

Supported, in part, by Intramural funding from the Jefferson College of Nursing.

Dr. Swan's institution received funding from the Jefferson College of Nursing intramural grant.

The remaining authors have disclosed that they do not have any potential conflicts of interest.

Address requests for reprints to: David A. Oxman, MD, FACP, Thomas Jefferson University,

David Oxman, 650 Walnut Street, Suite 650, Philadelphia, PA, 19106. E-mail:

david.oxman@jefferson.edu

\begin{abstract}
Objective: To compare usage patterns and outcomes of a nurse practitioner-staffed medical ICU and a resident-staffed physician medical ICU.

Design: Retrospective chart review of 1,157 medical ICU admissions from March 2012 to February 2013.

Setting: Large urban academic university hospital.

Subjects: One thousand one hundred fifty-seven consecutive medical ICU admissions including 221 nurse practitioner-staffed medical ICU admissions (19.1\%) and 936 resident-staffed medical ICU admissions (80.9\%).

Interventions: None.

Measurements and Main Results: Data obtained included age, gender, race, medical ICU admitting diagnosis, location at time of ICU transfer, code status at ICU admission, and severity of illness using both Acute Physiology and Chronic Health Evaluation II scores and a model for relative expected mortality. Primary outcomes compared included ICU mortality, in-hospital mortality, medical ICU length of stay, and post-ICU discharge hospital length of stay. Patients admitted to the nurse practitioner-staffed medical ICU were older (63 \pm 16.5 vs $59.2 \pm 16.9$ yr for resident-staffed medical ICU; $p=0.019)$, more likely to be transferred from an inpatient unit (52.0\% vs 40.0\% for the resident-staffed medical ICU; $p=0.002)$, and had a higher severity of illness by relative expected mortality $(21.3 \%$ vs $17.2 \%$ for the resident-staffed medical ICU; $p$ $=0.001)$. There were no differences among primary outcomes except for medical ICU length of stay (nurse practitioner-resident-staffed $7.9 \pm 7.5 d$ vs resident-staffed medical ICU $5.6 \pm 6.5 d$; $p=0.0001)$. Post-hospital discharge to nonhome location was also significantly higher in the nurse practitioner-ICU (31.7\% in nurse practitioner-staffed medical ICU vs $23.9 \%$ in residentstaffed medical ICU; $p=0.24$ ).
\end{abstract}


Conclusions: We found no difference in mortality between an nurse practitioner-staffed medical ICU and a resident-staffed physician medical ICU. Our study adds further evidence that advanced practice providers can render safe and effective ICU care.

Over the past few decades, the demand for critical care services (1) has increased dramatically and outstripped the supply of trained intensivists (2). In academic settings, the shortfall of the critical care workforce has been further complicated by work hour restrictions on trainees. Meanwhile, safety concerns related to the inadequate intensivist physician workforce have been expressed by national regulatory agencies, healthcare purchasers, and professional societies ( $\underline{3}-$ 5). In this context, patient care delivery models in critical care continue to evolve, including the use of advanced practice providers such as nurse practitioners (NPs) and physician assistants.

The operational approaches to incorporating advanced practice providers into intensive care settings vary widely. In some cases, advanced practice providers have been added to traditional physician staffing models, and in other cases, they have assumed duties traditionally performed by physicians $(\underline{6-11})$. Although NPs were first introduced to the healthcare workforce in the 1960 s $(12,13)$, the use of NPs as providers in the ICU is a relatively recent development that has expanded rapidly (14).

Yet despite the proliferation of NPs in the critical care workforce, studies examining outcomes in ICUs staffed by advanced practice providers with traditional models are limited. Recently, Costa et al (14) published a multicenter study comparing outcomes in ICUs in which advanced practice providers were part of the ICU team versus ICUs without advanced practice provider participation. Published data on outcomes in ICUs in which NPs completely replaced a part of the physician team (i.e., resident physicians) are limited to one study (ㄱ).

In January 2012, Thomas Jefferson University Hospital (TJUH) opened a new eight-bed medical ICU (MICU) staffed with NPs. The NPs were under the direct supervision of board-certified medical intensivists and could also consult at any time with critical care fellows. The hospital continued to operate another 17-bed MICU staffed by internal medicine residents, which was also under the supervision of critical attendings and fellows. In order to evaluate the safety and effectiveness of the new NP-ICU-staffing model at our institution, and to build on the existing body of literature on the subject, we examined usage patterns and outcomes between the two MICUs at TJUH.

\section{Methods}

Study Design

This was a retrospective review of ICU usage and outcomes of patients admitted to two different MICUs at our urban university hospital, TJUH. Patients were admitted either to a 17-bed internal medicine resident-staffed MICU (RES-MICU) or an eight-bed NP-staffed MICU (NPMICU). Daytime staffing consisted of two internal medicine residents and four interns in the RES-MICU and two NPs in the NP-ICU. Interns functioned under the direct supervision of residents who were either postgraduate year 2 or 3 . Nighttime coverage in the RES-MICU consisted of one resident and intern, while the NP-MICU was covered by one NP (Table 1). Both MICUs were supervised by an attending critical care physician during daytime hours. 
Additionally, a critical care fellow was in-house 24 hours a day, participated in morning rounds, and was available to assist residents or NPs as needed. The internal medicine interns and residents typically rotated through the MICU for a 1-month period. The NPs worked exclusively in the MICU and had the same clinical responsibilities as residents. However, the NPs had no other roles besides direct patient care, while the residents had educational responsibilities. During the study period, there were six full-time equivalent NPs. With the exception of one NP, all were new graduates of acute care nurse practitioner programs. Five of the six NPs had at least 2 years of bedside nursing experience. Both units were overseen by the same MICU physician director and nurse manager and used the same critical care nursing staff that rotated between both units. Triage decisions regarding which MICU an ICU patient would be admitted to were made by either the on-call critical care fellow or the attending physician. There were no official guidelines given to MICU attendings or fellows for deciding where to triage patients. However, the MICU director did recommend consideration of bed availability, bedside nursingpatient ratios, as well as the even distributions of admissions between admitting teams during the course of the day. Although no official data existed with regard to daily bed census, both units typically functioned near capacity.

\section{Data}

We used a previously created quality improvement database to retrospectively review all MICU admissions from March 2012 through February 2013. After removing duplicate admissions and cross-checking entries with University Health Consortium (UHC) data to verify accuracy we analyzed 1,157 admissions. The NP-MICU at TJUH began in January 2012, and we intentionally did not examine the first 2 months of data to allow for an adjustment period for the staff and operational workflow issues to be reconciled. Both units could accept admissions 24 hours a day. Patients admitted to one ICU and then transferred between units were grouped based on their original admission unit and their outcomes were analyzed on an intention-to-treat basis. We obtained data on patient characteristics including age, gender, race, MICU admitting diagnosis, location at time of ICU transfer, and code status at ICU admission. To assess severity of illness at ICU admission, we used the relative expected mortality based on risk-adjustment models for mortality from the UHC which provides data on all TJUH admissions. Additionally, Acute Physiology and Chronic Health Evaluation (APACHE) II scores from ICU admission were available on a random subset of patients. Finally, we collected data on MICU and hospital mortality, MICU discharge destination, MICU and hospital length of stay (LOS), and total hospital charges. Data were obtained from an MICU excel database tracking all MICU admissions that had been established as part of a previous MICU quality improvement activity, as well as from the hospital electronic medical record. All MICU admissions were then crossreferenced with the UHC to verify the accuracy of clinical information and ascertain information regarding hospital costs. The primary outcomes were ICU mortality, in-hospital mortality, ICU LOS, and post-ICU discharge hospital LOS. Secondary outcomes were hospital discharge location to post-acute care, MICU readmission rates within 48 hours of ICU discharge, and total observed hospital charges. 
Statistical Analysis

Percentages or means and SDs were used to describe patient characteristics in each of the two MICU patient treatment groups and nonparametric comparisons of the groups were conducted using Wilcoxon signed rank tests or chi-square tests as appropriate. The patient treatment groups were compared as to age, race, gender, facility at transfer, code status at transfer, severity score, and diagnosis at admission.

Two-block binary logistic, ordinal, and linear regression procedures employing the enter method were used to examine the effect of treatment in the NP-MICU versus the RES-MICU group on several outcomes: MICU mortality, hospital mortality, MICU readmission within 48 hours, posthospital discharge to a nonhome location or post-acute care setting (long-term acute care hospital [LTACH], skilled nursing facility [SNF], or acute or subacute rehabilitation), MICU LOS, hospital LOS, post-MICU discharge LOS, and charges observed. For all multivariate analyses, the demographic characteristics on which the patient treatment groups differed including age, expected mortality, and facility at transfer were entered into the first block of the design, whereas treatment group assignment was entered into the second block.

Database management and statistical analyses were performed using Excel (Microsoft, Redmond, WA) and SPSS, version 19.0 (IBM North America, New York, NY). The study was reviewed and approved by the Institutional Review Board (IRB) of Thomas Jefferson University. After IRB approval was obtained, data were collected retrospectively.

\section{Results}

\section{Patient Characteristics}

There were 1,157 patients admitted to an MICU at TJUH (Table 2) during the study period. Of these, 221 patients $(19.1 \%)$ were admitted to the MICU-NP and 936 [80.9\%] were admitted to the RES-MICU. Twenty patients $(1.7 \%)$ were transferred directly between the NP-MICU and the RES-MICU during the study period. There were differences in baseline characteristics of patients cared for in the two MICUs. Patients admitted to the NP-MICU were older $(63.3 \pm 16.5$ yr) when compared with the RES-MICU $(59.2 \pm 16.9 \mathrm{yr} ; \mathrm{p}=0.019)$. Location prior to transfer to the MICU varied between the two units with a higher likelihood that patients in the NPMICU were admitted from an inpatient area $(52.0 \%$ vs $40.0 \%$ in the RES-MICU; $p=0.002)$, while RES-MICU patients were more likely to be transferred from an outside hospital (20.6\% vs $14.5 \%$ NP-MICU) and the emergency department (29.9\% vs $37.4 \%$ NP-MICU). Risk of ICU death by APACHE II score did not differ significantly between units. However patients admitted to the NP-MICU had a higher risk of mortality by the UHC risk model $(21.3 \%$ vs $17.2 \% ; \mathrm{p}=0.001)$. There was no significant difference in gender, race, code status, or diagnosis at the time of MICU admission.

\section{Outcomes}

As indicated in Table 3, binary logistic regression procedures did not reveal any statistically significant differences between the NP-MICU and the RES-MICU patient groups in MICU 
mortality (14.5\% in NP-MICU vs $13.1 \%$ in RES-MICU; $p=0.441)$ or hospital discharge mortality (24.4\% in NP-MICU vs $24.8 \%$ in RES-MICU; $p=0.72)$. In that hospital discharge mortality was a dichotomous variable (expired vs other), we further examined hospital discharge using all available locations (home, rehab, LTACH, SNF, hospice, and expired), but the combined ordinal regression analysis did not uncover significant differences in our MICU treatment groups $(\mathrm{p}=0.678)$. Although unable to perform a priori power analysis, the number of patients in both treatment groups was large enough for sufficient power to exclude a clinical significance in mortality. The probability of a false-negative finding for MICU and hospital mortality is 0.09 and 0.04 , respectively.

Further examination using linear regression techniques comparing post-hospital discharge to nonhome location was significant (31.7\% in NP-MICU vs $23.9 \%$ in RES-MICU; $p=0.24$ ). The MICU patient treatment groups did not differ in terms of readmission to the MICU within 48 hours. Using linear regression techniques, we compared our MICU patient treatment groups on several LOS outcomes: MICU LOS, hospital LOS, and post-MICU discharge LOS. We observed a statistically significant difference between the NP-MICU and the RES-MICU patient treatment groups for MICU LOS $(\mathrm{t}=-4.1 ; \mathrm{p}=0.0001)$. Patients stayed in the MICU longer in the NPMICU group than in the RES-MICU $(\mathrm{M}=7.9 \mathrm{~d}, \mathrm{SD}=7.5 \mathrm{vs} \mathrm{M}=5.6 \mathrm{~d}, \mathrm{SD}=6.5$, respectively). No differences between the MICU treatment groups were found for hospital LOS or post-MICU discharge LOS. We also compared our treatment groups on hospital charges observed. However, we did not detect differences between the MICU treatment groups on this outcome.

\section{Discussion}

In our large urban academic university hospital, implementation of an NP-MICU created an opportunity to directly compare outcomes of two MICU staffing models without the use of historical controls. This retrospective review showed no difference in mortality between an NPMICU and a traditional resident-staffed physician MICU. These findings support previously published results suggesting that NP-MICUs provide safe models of care delivery and are reasonable staffing alternatives to traditional physician-only models ().

To date, past findings only investigated traditional outcomes including LOS, mortality, and posthospital discharge location. Our study adds to the notion that NPs provide high-quality and costeffective care through comparing additional variables including post-MICU discharge LOS, MICU readmission rates, and charge observed.

Patients in our study admitted to the NP-MICU did have a significantly longer ICU LOS. Although this could suggest differences in clinical practice between NPs and medical residents, it is more likely a reflection of different triage patterns between the two units. Patients admitted to the NP-MICU were older and less likely to be admitted from the emergency department. This suggests that, at least during the study period, more chronically and critically ill patients were triaged to the NP-MICU than the RES-MICU. These findings are congruent with Gershengorn et al () in which an advanced practice provider-staffed MICU received patients who were also older, more likely to come from the hospital floor, and more frequently had a do-not-resuscitate order at the time of ICU admission. Additionally, the increased LOS may be attributable to discharge location, as coordinating transfer to an outside facility may delay ICU discharge 
significantly. Patients cared for in the NP-MICU were more likely to be discharged to an LTACH, SNF, or acute or subacute rehabilitation when compared with patients in the RESMICU. Whether these differences would have persisted if the study period was longer is uncertain. It is plausible that MICU LOS differed due to variation in discharge planning and practices among the two group of providers. Although direct comparison of discharge practices of physicians and advanced practice providers has not been identified, the complexities and variation of discharging patients from the critical care setting have recently been reported ( 15 , 16). Interestingly, despite the longer ICU LOS in the NP-MICU, overall hospital charges did not differ significantly compared with the RES-MICU, suggesting that use of resources remained similar between the two populations. This finding supports previously published work that NPs are cost-effective providers $(\underline{6}, \underline{7})$.

This study had several limitations. First, it is a single-center study conducted at a large urban academic university hospital. The results therefore may not be generalizable to other hospitals, particularly those in which there is no 24-hour-a-day critical care attending or fellow physician available. However, the primary measure of safe care in our study was mortality, and both units in the study had mortality rates similar to national estimates of mortality (12-16\%) in patients with an ICU admission $(\underline{17}, \underline{18})$.

The study is a retrospective review and not a prospective randomized control trial. Therefore, we cannot completely control for differences in the patient population of the two ICUs. Finally, we used administrative data for risk of mortality with only a subset having a validated predictor of ICU mortality (APACHE II) calculated. We were unable to complete a comparison to national data because this was a modest single-center study comparing outcomes solely at our institution.

\section{Conclusions}

Our study adds further evidence to the idea that advanced practice providers can render safe and effective ICU care. The findings support the growing role for these providers in the care of ICU patients. More study is needed to investigate possible differences in clinical practice between physician and advanced practice providers in the ICU as well as to explore ways to optimize their use in expanding the critical care workforce.

\section{References}

1. Halpern NA, Pastores SM: Critical care medicine in the United States 2000-2005: An analysis of bed numbers, occupancy rates, payer mix, and costs. Crit Care Med 2010; 38:65-71 Ovid Full Text Bibliographic Links [Context Link]

2. Angus DC, Kelley MA, Schmitz RJ, et al; Committee on Manpower for Pulmonary and

Critical Care Societies (COMPACCS): Caring for the critically ill patient. Current and projected workforce requirements for care of the critically ill and patients with pulmonary disease: Can we meet the requirements of an aging population? JAMA 2000; 284:2762-2770 SerialsSolutions Bibliographic Links [Context Link] 
3. Ewart GW, Marcus L, Gaba MM, et al: The critical care medicine crisis: A call for federal action: A white paper from the critical care professional societies. Chest 2004; 125:1518-1521 Ovid Full Text Bibliographic Links [Context Link]

4. Landsperger JS, Williams KJ, Hellervik SM, et al: Implementation of a medical intensive care unit acute-care nurse practitioner service. Hosp Pract (1995) 2011; 39:32-39 [Context Link]

5. The Leapfrog Group: Leapfrog. 2015. Available at: http://www.leapfroggroup.org. Accessed June 5, 2015 [Context Link]

6. Burns SM, Earven S, Fisher C, et al; University of Virginia Long Term Mechanical

Ventilation Team: Implementation of an institutional program to improve clinical and financial outcomes of mechanically ventilated patients: One-year outcomes and lessons learned. Crit Care Med 2003; 31:2752-2763 Ovid Full Text Bibliographic Links [Context Link]

7. Gershengorn HB, Wunsch H, Wahab R, et al: Impact of nonphysician staffing on outcomes in a medical ICU. Chest 2011; 139:1347-1353 SerialsSolutions Bibliographic Links [Context Link]

8. Gracias VH, Sicoutris CP, Stawicki SP, et al: Critical care nurse practitioners improve compliance with clinical practice guidelines in "semiclosed" surgical intensive care unit. J Nurs Care Qual 2008; 23:338-344 Ovid Full Text Bibliographic Links [Context Link]

9. Hoffman LA, Tasota FJ, Zullo TG, et al: Outcomes of care managed by an acute care nurse practitioner/attending physician team in a subacute medical intensive care unit. Am J Crit Care 2005; 14:121-130; quiz 131 SerialsSolutions Bibliographic Links [Context Link]

10. Hoffman LA, Miller TH, Zullo TG, et al: Comparison of 2 models for managing tracheotomized patients in a subacute medical intensive care unit. Respir Care 2006; 51:12301236 SerialsSolutions Bibliographic Links [Context Link]

11. Russell D, VorderBruegge M, Burns SM: Effect of an outcomes-managed approach to care of neuroscience patients by acute care nurse practitioners. Am J Crit Care 2002; 11:353-362 SerialsSolutions Bibliographic Links [Context Link]

12. Jastremski CA: Nonphysician clinicians in the ICU. Semin Respir Crit Care Med 2001; 22:89-94 SerialsSolutions Bibliographic Links [Context Link]

13. Kleinpell RM, Ely EW, Grabenkort R: Nurse practitioners and physician assistants in the intensive care unit: An evidence-based review. Crit Care Med 2008; 36:2888-2897 Ovid Full Text Bibliographic Links [Context Link]

14. Costa DK, Wallace DJ, Barnato AE, et al: Nurse practitioner/physician assistant staffing and critical care mortality. Chest 2014; 146:1566-1573 SerialsSolutions Bibliographic Links [Context Link]

15. Heidegger CP, Treggiari MM, Romand JA; Swiss ICU Network: A nationwide survey of intensive care unit discharge practices. Intensive Care Med 2005; 31:1676-1682 SerialsSolutions Bibliographic Links [Context Link]

16. Stelfox HT, Lane D, Boyd JM, et al: A scoping review of patient discharge from intensive care: Opportunities and tools to improve care. Chest 2015; 147:317-327 SerialsSolutions Bibliographic Links [Context Link]

17. Zimmerman JE, Kramer AA, Knaus WA: Changes in hospital mortality for United States intensive care unit admissions from 1988 to 2012. Crit Care 2013; 17:R8 SerialsSolutions [Context Link] 
18. Young MP, Birkmeyer JD: Potential reduction in mortality rates using an intensivist model to manage intensive care units. Eff Clin Pract 2000; 3:284-289 SerialsSolutions Bibliographic Links [Context Link]

KEYWORDS: advanced practice provider; critical care workforce; intensive care; nonphysician provider; nurse practitioner 